\title{
Performance Analysis of Asymmetric PCMA Signal Demodulation
}

\author{
Xingchen $\mathrm{Xu}^{\mathrm{a}}$, Jian Cheng ${ }^{\mathrm{b},{ }^{*}}$ and Jingyu Tang ${ }^{\mathrm{c}}$ \\ Army Engineering University of PLA, China. \\ atammyxxc@163.com, *, b chjatice@163.com, cmrtangjingyu@qq.com
}

Keywords: modern apprenticeship, stakeholders, guarantee mechanism.

\begin{abstract}
Targeting at the demodulation of asymmetric Paired Carry Multiple Access (PCMA) signal mixed by primary and secondary signal, this paper has studied the implementation framework of demodulation. First the frequency offset and phase of QPSK modulated PCMA signal are precisely locked by phase lock loop. Meanwhile, the delay and amplitude of primary signal are respectively estimated by conjugate correlation method and quartic method. After four parameters are obtained, the interference signal is reconstructed to obtain the secondary signal after canceling the mixed signal. And the error rates under different error conditions are deduced and simulated. The impacts of each parameter error on the performance of demodulation are obtained through a comparative analysis.
\end{abstract}

\section{Introduction}

In 1998, Mark Danker [1] from Via Sat Company in America proposed a two-way satellite communication technology utilizing Paired Carrier Multiple Access (PCMA) and based on transparent transponder, and he also applied self-interference cancellation technology [2]. This technology improves the utilization of satellite channel and frequency band and affects the demodulation of signal when saving the bandwidth resources. The ground master receives the downlink mixed signal formed by the aliasing of the local signal and the terminal signal of the small station. The local master knows the locally transmitted uplink signal precisely and thereby it can cancel the self-interfering signal from the received mixed signal, so that the terminal signal of the small station can be correctly recovered. The main content of this paper is divided into three parts: the first part is to establish the implementation framework of PCMA signal demodulation in asymmetric mode; the second part conducts simulation and analyzes the impacts of each parameter error on the performance of demodulation; the third part is a summary of this study.

\section{Model of Asymmetric PCMA System}

Asymmetric PCMA system is a self-interfering system that can be demodulated only after cancelling the self-interfering signal, so as to realize signal separation. The diagram of the demodulation model of asymmetric PCMA system is shown in Figure 1, with four parameters to be estimated, namely delay $\tau$, carrier frequency $f$, carrier phase $\theta$ and amplitude $h$.

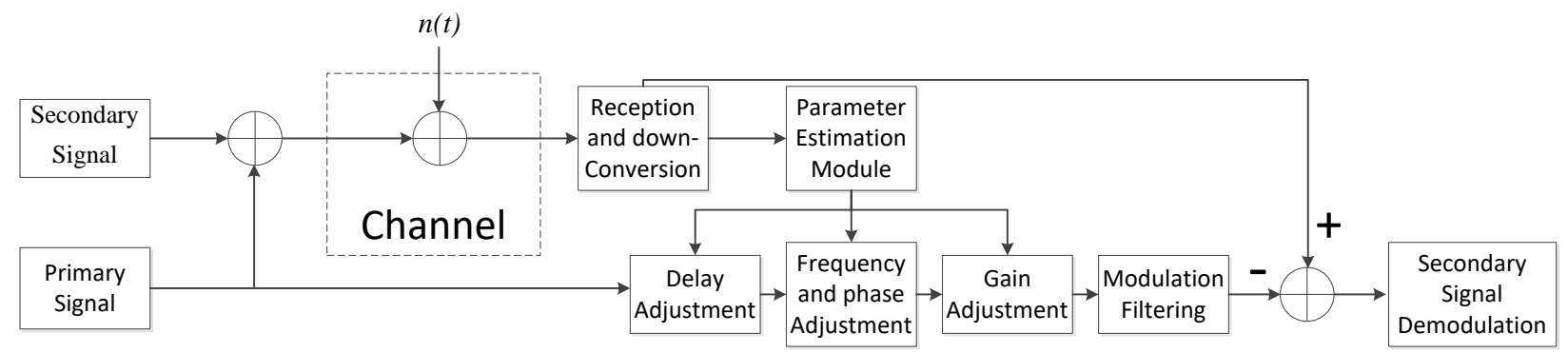

Figure 1. Diagram of the demodulation model of asymmetric PCMA system

Asymmetric PCMA signal is formed by the aliasing of the primary and the secondary signal. In order to facilitate the analysis, this study assumes that the channel is Gaussian channel [3], and the signal received through matched filtering by the channel is: 


$$
y(t)=s_{1}(t)+s_{2}(t)+n(t)
$$

Thereunto, $s_{1}(t)$ is the primary signal; $s_{2}(t)$ is the secondary signal; $n(t)$ is the Gaussian white noise which is introduced through the channel; the unilateral power spectral density (PSD) is $N_{0}$. The complex baseband model of PCMA received signal can be expressed as:

$$
y(t)=h_{1}(t) e^{j\left(2 \pi \Delta f_{1}(t) t+\theta_{1}(t)\right)} x_{1}(t)+h_{2}(t) e^{j\left(2 \pi \Delta f_{2}(t) t+\theta_{2}(t)\right)} x_{2}(t)+n(t)
$$

Thereunto, $h_{1}(t)$ and $h_{2}(t)$ are the instantaneous amplitudes of the two channels when receiving signals; $f_{1}(t)$ and $f_{2}(t)$ represent the instantaneous frequency offsets of the two signals; $\theta_{1}(t)$ and $\theta_{2}(t)$ represent the phase offsets of the two signals; $n(t)$ is the instantaneous noise; $x_{1}(t)$ and $x_{2}(t)$ are two digital baseband modulated signals. This chapter uses QPSK modulation [4], whose expression is:

$$
\begin{array}{r}
x_{1}(t)=\sum_{m=-\infty}^{\infty} a_{1, m} g_{1}\left(t-m T_{1}+\tau_{1}(t)\right) \\
x_{2}(t)=\sum_{n=-\infty}^{\infty} a_{2, n} g_{2}\left(t-n T_{2}+\tau_{2}(t)\right)
\end{array}
$$

In equations (3) and (4), $a_{1, m}$ represents the $\mathrm{M}^{\mathrm{Th}}$ symbol transmitted by the first channel, and $a_{2, n}$ represents the $\mathrm{n}^{\text {th }}$ symbol transmitted by the second channel, which are independent and identically distributed; $g_{1}(t)$ and $g_{2}(t)$ the two signals are the equivalent channel filters of two signals respectively, including roll-forming filters, channel filters and matched filters; $\tau_{1}(t)$ and $\tau_{2}(t)$ are the instantaneous delays of two signals respectively. The study takes the channel parameters in a certain period of time as constants, and then the complex baseband model of signal received becomes:

$$
y(t)=h_{1} e^{j\left(2 \pi \Delta f_{1} t+\theta_{1}\right)} x_{1}(t)+h_{2} e^{j\left(2 \pi \Delta f_{2} t+\theta_{2}\right)} x_{2}(t)+n(t)
$$

So $x_{1}(t)=\sum_{m=-\infty}^{\infty} a_{1, m} g_{1}\left(t-m T_{1}+\tau_{1}\right), x_{2}(t)=\sum_{n=-\infty}^{\infty} a_{2, n} g_{2}\left(t-n T_{2}+\tau_{2}\right)$. In order to reconstruct the self-interfering signal $s_{1}(t)$, it is necessary to estimate the transmission delay, the carrier frequency and phase, and the signal amplitude of the self-interfering signal. The reconstructed self-interfering signal can be expressed as:

$$
\hat{s}_{1}(t)=\hat{h}_{1} e^{j\left(2 \pi \Delta \hat{f}_{1}+\hat{\theta}_{1}\right)} \hat{x}_{1}(t)=\hat{h}_{1} e^{j\left(2 \pi \Delta \hat{f}_{1}+\hat{\theta}_{1}\right)} \sum_{m=-\infty}^{\infty} a_{1, m} \hat{g}_{1}\left(t-m T_{1}+\hat{\tau}_{1}\right)
$$

In the formula, $\Delta \hat{f}_{1}, \hat{\theta}_{1}, \hat{\tau}_{1}$ and $\hat{h}_{1}$ are the estimations of carrier frequency, carrier phase, and transmission delay and signal amplitude of the self-interfering signal respectively. The demodulation performance is related to the errors of the above estimations of parameters.

\section{Simulation Analysis}

In the parameter estimation, the carrier frequency of the signal is estimated by using a phase-locked loop for real-time tracking. Generally speaking, there is almost no error after the frequency is averaged, so no specific analysis will follow. The rest of parameters are carrier phase, delay and amplitude of the signal, respectively. After theoretical analysis, the simulation results verify that the impact of each parameter error on bit error rate conforms to the theoretical derivation.

\subsection{Analysis of Phase Error.}

Formula derivation. Suppose the received complex signal is: 


$$
\begin{aligned}
y(t) & =s_{1}(t)+s_{2}(t)+n(t) \\
& =h_{1} e^{j\left(2 \pi f_{c} t+\theta_{1}\right)} x_{1}(t)+h_{2} e^{j\left(2 \pi f_{c} t+\theta_{2}\right)} x_{2}(t)+n(t)
\end{aligned}
$$

Among them $s_{1}(t)=h_{1} e^{j\left(2 \pi f_{c} t+\theta_{1}\right)} x_{1}(t)$ is the primary signal, $s_{2}(t)=h_{2} e^{j\left(2 \pi f_{c} t+\theta_{2}\right)} x_{2}(t)$ is the secondary signal, $h_{1}, h_{2}$ are the signal amplitudes, $n(t)$ is the white noise of which the variance is $\sigma^{2}$. The reconstructed signal of the primary signal is:

$$
\hat{s}_{1}(t)=h_{1} e^{j\left(2 \pi f_{c} t+\theta_{1}+\Delta \theta\right)} x_{1}(t)
$$

Thereunto $\Delta \theta$ is the phase error. Conduct the operation of (7) - (8), and we can get:

$$
\begin{aligned}
y(t)-\hat{s}_{1}(t) & =s_{1}(t)+s_{2}(t)+n(t)-\hat{s}_{1}(t) \\
& =h_{1} e^{j\left(2 \pi f_{c} t+\theta_{1}\right)} x_{1}(t)+h_{2} e^{j\left(2 \pi f_{c} t+\theta_{2}\right)} x_{2}(t)-h_{1} e^{j\left(2 \pi f_{c} t+\theta_{1}+\Delta \theta\right)} x_{1}(t)+n(t) \\
& =h_{1} e^{j\left(2 \pi f_{c} t+\theta_{1}\right)} x_{1}(t)\left(1-e^{j \Delta \theta}\right)+h_{2} e^{j\left(2 \pi f_{c} t+\theta_{2}\right)} x_{2}(t)+n(t) \\
& =h_{1} \sqrt{2-2 \cos \Delta \theta} e^{j\left(2 \pi f_{c} t+\theta_{1}+\alpha\right)} x_{1}(t)+h_{2} e^{j\left(2 \pi f_{c} t+\theta_{2}\right)} x_{2}(t)+n(t)
\end{aligned}
$$

Among them, $\cos \alpha=\frac{1-\cos \Delta \theta}{\sqrt{2-2 \cos \Delta \theta}}, \sin \alpha=\frac{-\sin \Delta \theta}{\sqrt{2-2 \cos \Delta \theta}}$. In the case of small phase error, $h_{1} \sqrt{2-2 \cos \Delta \theta} \square h_{2}$, and then the error $h_{1} \sqrt{2-2 \cos \Delta \theta} e^{j\left(2 \pi f_{c} t+\theta_{1}+\alpha\right)} x_{1}(t)$ obtained after offsetting the self-interfering signal can be regarded as noise ${ }^{[5]}$. The signal to noise ratio of the signal $y(t)-\hat{s}_{1}(t)$ is:

$$
s n r=\frac{h_{2}^{2}}{h_{1}^{2}(2-2 \cos \Delta \theta)+\sigma^{2}}
$$

Through the formula of QPSK signal error rate, we can get the relation between the phase error $\Delta \theta$ and bit error rate:

$$
P_{e_{-} \Delta \theta}=\frac{1}{2} \operatorname{erfc}\left\{\sqrt{\frac{h_{2}^{2}}{2\left[h_{1}^{2}(2-2 \cos \Delta \theta)+\sigma^{2}\right]}}\right\}
$$

Simulation verification. The parameter settings of phase error simulation are given by Table 1 .

\begin{tabular}{|c|c|c|}
\hline$h_{1}=10$ & $h_{2}=1$ & $\sigma^{2}=0.09$ \\
\hline$\Delta \theta$ & $h_{1}^{2}(2-2 \cos \Delta \theta)$ & $s n r(\mathrm{~dB})$ \\
\hline$\pi / 1000$ & $9.8696 \mathrm{e}-04$ & 10.4102 \\
\hline$\pi / 200$ & 0.0247 & 9.4054 \\
\hline$\pi / 100$ & 0.0987 & 7.2426 \\
\hline$\pi / 70$ & 0.2014 & 5.3553 \\
\hline$\pi / 50$ & 0.3947 & 3.1457 \\
\hline
\end{tabular}
Simulation results are shown in Figure 2. When the signal-to-noise ratio is large, the simulation value is basically consistent with the theoretical value. When the signal-to-noise ratio is small, the bit error rate obtained by the simulation is slightly less than the theoretical value. It can be explained as follows: from equation (9), it can be seen that after the cancellation by utilizing the reconstructed signal which has phase error, the residual part $h_{1} \sqrt{2-2 \cos \Delta \theta} e^{j\left(2 \pi f_{c} t+\theta_{1}+\alpha\right)} x_{1}(t)$ is actually a regular signal waveform. Compared to the noise of the same power, the residual signal has less impact on the bit error rate of the demodulation of the secondary signal.

Table 1 Parameter settings of phase error simulation 


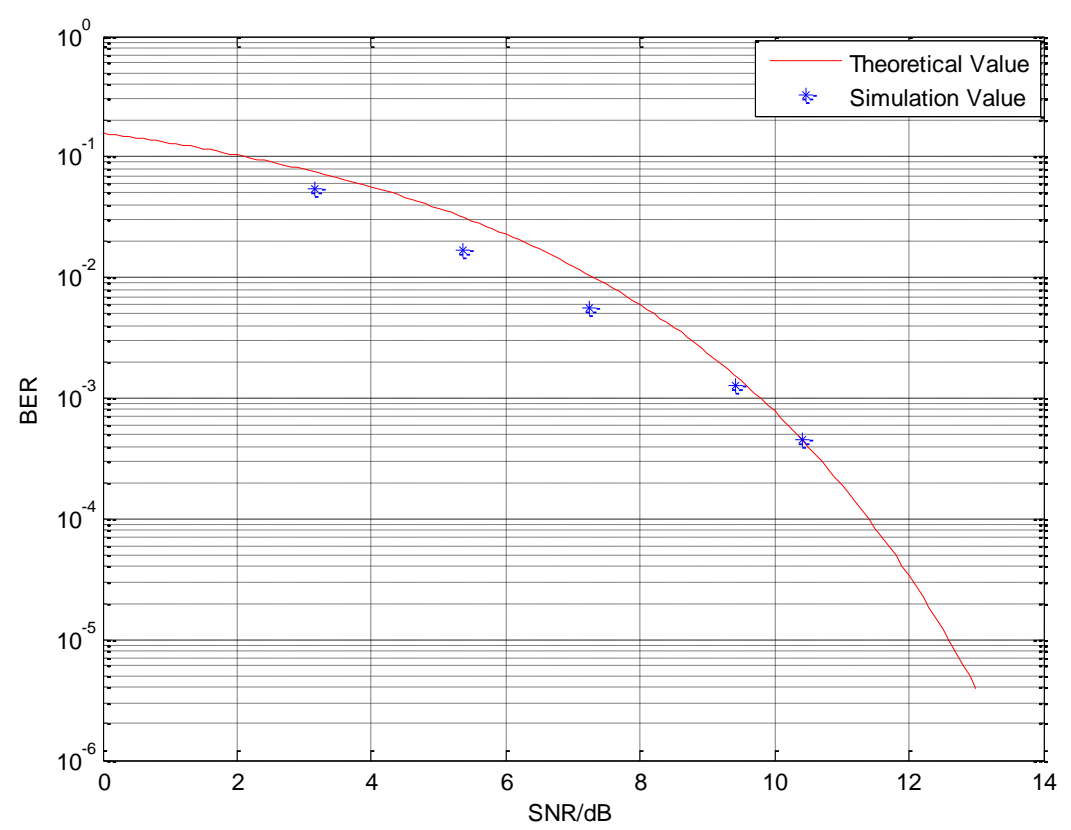

\subsection{Analysis of Delay Error.}

Figure 2. Diagram of phase error simulation

Suppose the received signal is the signal shown in Eq. (7), and the reconstructed signal of self-interfering signal is:

$$
\hat{s}_{1}(t)=h_{1} e^{j\left(2 \pi f_{c} t+\theta_{1}\right)} x_{1}(t-\tau)
$$

Among them $\tau$ is the delay error. Conduct the operation of (7) - (12), and we can get:

$$
\begin{aligned}
y(t)-\hat{s}_{1}(t) & =s_{1}(t)+s_{2}(t)+n(t)-\hat{s}_{1}(t) \\
& =h_{1} e^{j\left(2 \pi f_{c} t+\theta_{1}\right)} x_{1}(t)+h_{2} e^{j\left(2 \pi f_{c} t+\theta_{2}\right)} x_{2}(t)-h_{1} e^{j\left(2 \pi f_{c} t+\theta_{1}\right)} x_{1}(t-\tau)+n(t) \\
& =h_{1} e^{j\left(2 \pi f_{c} t+\theta_{1}\right)}\left[x_{1}(t)-x_{1}(t-\tau)\right]+h_{2} e^{j\left(2 \pi f_{c} t+\theta_{2}\right)} x_{2}(t)+n(t)
\end{aligned}
$$

As can be seen from Eq. (13), when there is a delay error, many pulses of width $\tau$ will appear when the self-interfering signal is canceled, as shown in Fig.3. If the location of the pulse happens to be the location of the sampling decision when the useful signal is demodulated, a larger bit error rate will result.

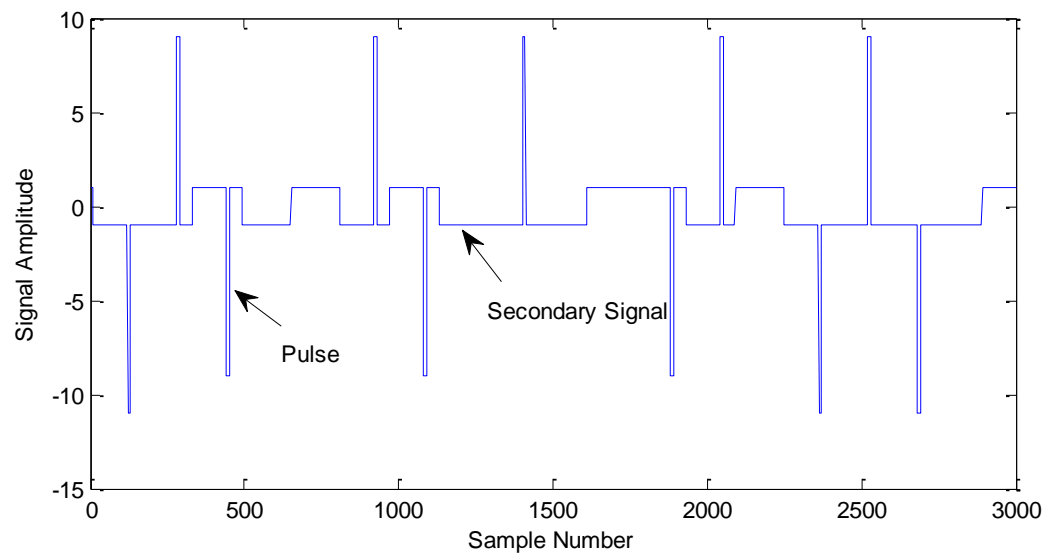

Figure 3. Effect of delay error on signal demodulation

\subsection{Analysis of Amplitude Error.}

Formula derivation. Suppose the received signal is the signal shown in Eq. (7), and the reconstructed signal of self-interference signal is:

$$
\hat{s}_{1}(t)=\left(h_{1}+\Delta h\right) e^{j\left(2 \pi f_{c} t+\theta_{1}\right)} x_{1}(t)
$$

Thereunto $\Delta A$ is the amplitude error. Conduct the operation of (7) - (14), and we can get: 


$$
\begin{aligned}
y(t)-\hat{s}_{1}(t) & =s_{1}(t)+s_{2}(t)+n(t)-\hat{s}_{1}(t) \\
& =h_{1} e^{j\left(2 \pi f_{c} t+\theta_{1}\right)} x_{1}(t)+h_{2} e^{j\left(2 \pi f_{c} t+\theta_{2}\right)} x_{2}(t)-\left(h_{1}+\Delta h\right) e^{j\left(2 \pi f_{c} t+\theta_{1}\right)} x_{1}(t)+n(t) \\
& =h_{2} e^{j\left(2 \pi f_{c} t+\theta_{2}\right)} x_{2}(t)-\Delta h e^{j\left(2 \pi f_{c} t+\theta_{1}\right)} x_{1}(t)+n(t)
\end{aligned}
$$

When $\Delta h \square h_{2}$, regard the error $\Delta h e^{j\left(2 \pi f_{c} t+\theta_{1}\right)} x_{1}(t)$ after canceling the self-interfering signal as noise [6]. The signal-to-noise ratio of the signal $y(t)-\hat{s}_{1}(t)$ is:

$$
s n r=\frac{h_{2}^{2}}{\Delta h^{2}+\sigma^{2}}
$$

Through the formula of QPSK signal error rate, we can get the relation between amplitude error $\Delta A$ and bit error rate:

$$
P_{e_{-} \Delta h}=\frac{1}{2} \operatorname{erfc}\left\{\sqrt{\frac{h_{2}^{2}}{2\left[\Delta h^{2}+\sigma^{2}\right]}}\right\}
$$

Simulation verification. The parameter settings of amplitude error simulation are given by Table 2 . Simulation results are shown in Figure 4. The simulated value is basically the same as the theoretical value when the signal-to-noise ratio is relatively large. When the signal-to-noise ratio is small, the bit error rate obtained by the simulation is slightly smaller than the theoretical value, the reason for which is similar to that in the analysis of phase error.

Table 2 Parameter settings of amplitude error simulation

\begin{tabular}{c|cc}
\hline$h_{1}=10$ & $h_{2}=1$ & $\sigma^{2}=0.09$ \\
\hline$\Delta h$ & & $s n r(\mathrm{~dB})$ \\
\hline 0.05 & 10.3386 \\
0.1 & 10 \\
0.2 & 8.8606 \\
0.3 & 7.4473 \\
0.4 & 6.0206 \\
0.5 & 4.6852 \\
\hline
\end{tabular}

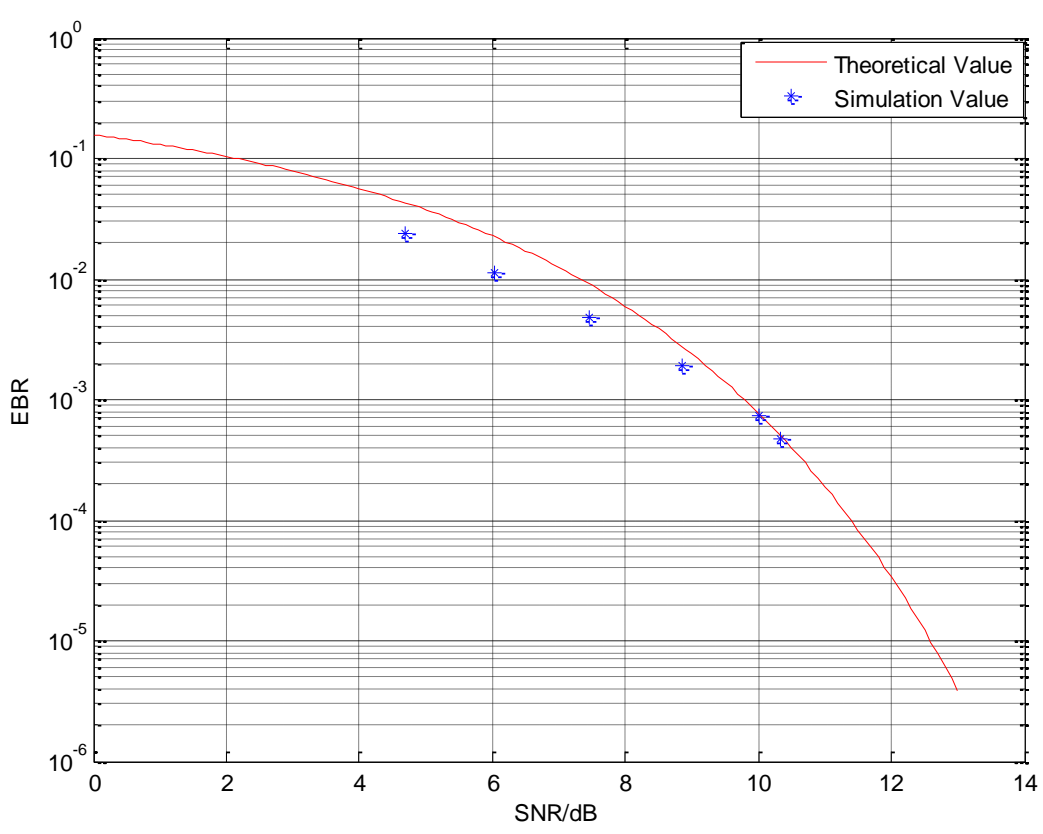

Figure 4. Diagram of amplitude error simulation 


\section{Conclusion}

PCMA signal can not only improve the utilization of frequency band of satellite channels and save bandwidth resources, but it can also have high anti-interception performance, which is of great significance to satellite communications. In this paper, the implementation framework of the demodulation system is established, and the impact of each parameter error on the demodulation performance and the corresponding causes are analyzed through theoretical derivation and simulation verification. Therefore, it is of certain reference value to the engineering application of PCMA signal. However, due to the limitation of algorithm research, the estimation algorithm used in this paper is not yet the optimal algorithm and thus needs to be further improved.

\section{Acknowledgments}

First and for the most, I would like to thank Prof. Cheng Jain for his careful guidance to me during my studies. At the same time, I also thank my senior fellow apprentice Tang Jingly for helping me. Last but not least, I want to thank my parents and my best friend Cui Xiao for their supporting for my study life.

\section{References}

[1]. Mark D. Paired carrier multiple access (PCMA) for satellite communications [A]. In: Pacific Telecommunications Conference[C], Honolulu Hawaii, Jan. 13, 1998: 787-791.

[2]. Danker M D, Miller M G, Mulligan M G. Self-interference cancellation for two-party relayed communication. US: Patent No. 5596439[P]. 1997-1-21.

[3]. GE Lei, Study on Technology of Separation and Demodulation for PCMA Signals [J].Radio Engineering, 2015, 45(08):39-42.

[4]. ZHAO Aiming, SUN Shirley, OUYANG Ming. Research and Implementation of QPSK Carrier Synchronization Algorithm Based on FPGA [J].TV Technology, 36(11):100-103.

[5]. YU Kayoing, LI Jing fang, PAN Shenmue. Impact Analysis of Carrier Recovery Error on Demodulation Performance [J]. Radio Communications Technology, 2014, 40(1):26-27, 40.

[6]. GUO Liming. Research on Key Technologies for Blind Demodulation of PCMA Signals [D].Zhengzhou: PLA Information Engineering University, 2015. 